\title{
TELEMEDICINE APPLICATIONS IN DERMATOLOGY
}

\author{
Hamidreza Tadayon ${ }^{1}$, Farzaneh Baniasadidamaski ${ }^{2 *}$
}

x1: faculty member of health information technology, Torbat heydariyeh University of medical sciences, Torbat heydariyeh, Iran.

2: student of health information technology, Torbat heydariyeh University of medical sciences, Torbat heydariyeh, Iran.

Correspondence:

Tel: +989377445991, E-mail: baniasadi72@gmail.com

\section{TYPE OF ARTICLE: CONFERENCE ABSTRACT}

\begin{abstract}
Introduction: One of the greatest challenges facing the health care system is to provide quality of care to a large segment of the population, which does not have access to specialty physicians in order to factors such as geographic limitations or socioeconomic conditions. The use of technology to deliver health care from a distance, or telemedicine, has been demonstrated as an effective way of overcoming certain barriers to care, particularly for communities located in rural and remote areas. Teledermatology, as one of the telemedicine applications, is used in the diagnosis and treatment of skin diseases.

Methods: This article was a reviewed study. The necessary data were gathered by reliable and relevant library and Internet resources. Data of online databases were gathered from the following databases: Google Scholar, SID, Pubmed, Sciencedirect. Collected data were summarized and analyzed by drawing comparative tables, and we finally determined similarities and differences aspects.

Results: Over the past decade, telemedicine services, particularly for skin diseases, have seen significant progress and has become a part of routine medical services. In Iran, although telemedicine services have not been used in practice, a few relevant research projects have been conducted. Teledermatology in Australia holds promise as an alternative means of delivering health care. Equipment is becoming financially accessible, easier to use and more widely available for health care workers and patients alike.

Conclusion: Most of the researchers and experts agree with the effectiveness of teledermatology for providing professional health care services and increasing the accessibility of these services; this method is not the final solution to overcome all difficulties related to the shortage of human and financial resources in health care systems. It should be considered as complementary to resolve some problems in providing high-quality health care.
\end{abstract}

KEYWORDS: Telemedicine, Teleconsultation, Skin diseases, Teledermatology

\section{Abstracts of First National Congress of Medical Informatics, Mashhad, Iran, February 2017}

(C) 2017 The Authors. This is an open access article under the terms of the Creative Commons Attribution-NonCommercialNoDerivs License, which permits use and distribution in any medium, provided the original work is properly cited, the use is non-commercial and no modifications or adaptations are made. 